\title{
QCD corrections to vector boson pair production in gluon fusion including interference effects with off-shell Higgs at the LHC
}

\author{
Fabrizio Caola, ${ }^{a}$ Matthew Dowling, ${ }^{b}$ Kirill Melnikov, ${ }^{b}$ Raoul Röntsch ${ }^{b}$ and \\ Lorenzo Tancredi ${ }^{b}$ \\ ${ }^{a}$ CERN Theory Division, \\ Geneva 23, CH-1211 Switzerland \\ ${ }^{b}$ Institute for Theoretical Particle Physics, KIT, \\ Karlsruhe, Germany \\ E-mail: fabrizio.caola@cern.ch, matthew.dowling@kit.edu, \\ kirill.melnikov@kit.edu, raoul.roentsch@kit.edu, \\ lorenzo.tancredi@kit.edu
}

ABSTRACT: We compute next-to-leading order (NLO) QCD corrections to the production of two massive electroweak bosons in gluon fusion. We consider both the prompt production process $g g \rightarrow V V$ and the production mediated by an exchange of an $s$-channel Higgs boson, $g g \rightarrow H^{*} \rightarrow V V$. We include final states with both on- and off-shell vector bosons with leptonic decays. The gluonic production of vector bosons is a loop-induced process, including both massless and massive quarks in the loop. For $g g \rightarrow Z Z$ production, we obtain the NLO QCD corrections to the massive loops through an expansion around the heavy top limit. This approximation is valid below the top production threshold, giving a broad range of invariant masses between the Higgs production and the top production thresholds in which our results are valid. We explore the NLO QCD effects in $g g \rightarrow Z Z$ focusing, in particular, on the interference between prompt and Higgs-mediated processes. We find that the QCD corrections to the interference are large and similar in size to the corrections to both the signal and the background processes. At the same time, we observe that corrections to the interference change rapidly with the four-lepton invariant mass in the region around the $Z Z$ production threshold. We also study the interference effects in $g g \rightarrow W^{+} W^{-}$production where, due to technical limitations, we only consider contributions of massless loops. We find that the QCD corrections to the interference in this case are somewhat larger than those for either the signal or the background.

KeYwords: NLO Computations, QCD Phenomenology

ARXIV EPRINT: 1605.04610 


\section{Contents}

1 Introduction 1

$2 \quad Z Z$ production 3

2.1 Details of the calculation 3

2.2 LHC phenomenology 8

$\begin{array}{lll}3 & W W \text { production } & 11\end{array}$

4 Conclusion $\quad 14$

\section{Introduction}

After the discovery of the Higgs boson during Run I at the Large Hadron Collider (LHC) [1, 2] an important task for Run II is a thorough study of its properties. In the Standard Model (SM), the Higgs field is solely responsible for the phenomenon of electroweak symmetry breaking (EWSB) that provides masses to fermions and weak gauge bosons in a consistent way. This minimal version of the EWSB mechanism predicts a stringent relation between masses of elementary particles and their couplings to the Higgs boson. Experimental studies of Higgs couplings to other Standard Model particles provide a direct test of this mechanism; any deviation from the minimal relation between couplings and masses will imply that the SM version of the EWSB mechanism is incomplete.

Measurements of the couplings are typically performed for the on-shell production and decay of the Higgs bosons, simply because the absolute majority of the Higgs bosons at the LHC are produced on-shell. However, it was recently realized that the off-shell production of the Higgs boson can also provide useful insights into its properties. Indeed, despite the extremely narrow width of the Higgs boson, the off-shell region is well-populated, with about one out of ten Higgs boson events in the $g g \rightarrow H \rightarrow Z Z$ process having an invariant mass of the two $Z$-bosons above $180 \mathrm{GeV}$ [3]. Furthermore, the interference between Higgsmediated amplitude $g g \rightarrow H \rightarrow V V$ and the prompt production amplitude $g g \rightarrow V V$ is strong and destructive in the high invariant mass region. This is expected since Higgs boson exchanges are supposed to unitarize the scattering amplitudes of massive fermions and gauge bosons. The observation of this unitarization effect at large values of $m_{Z Z}$ will be an important confirmation of the fact that the discovered Higgs boson is indeed the only agent of electroweak symmetry breaking mechanism, as predicted in the Standard Model.

Measurements in the off-shell region are also useful for another reason. Indeed, extraction of the Higgs couplings from the on-shell measurements is, in principle, compromised by the unknown value of the Higgs width, leading to an infinite-fold degeneracy in the extracted values of the couplings. It was pointed out by two of us [4] that this ambiguity is lifted by off-shell measurements, which are sensitive to the Higgs couplings only. The ratio 
of the off-shell to on-shell cross sections $p p \rightarrow H \rightarrow Z Z$ can then be used to obtain stringent constraints on the Higgs boson width [4-6]. Subsequent experimental analyses used this method to constrain the Higgs boson width to be less than a few times its Standard Model value [7-9]. This is to be compared with the direct constraints on the Higgs width that can be obtained from fitting the invariant mass distributions of four leptons or a photon pair around the Higgs mass. Because of detector resolution, such direct constraints on $\Gamma_{H}$ cannot probe values smaller than $\Gamma_{H} \sim 1 \mathrm{GeV}$, so they are about two orders of magnitude weaker than the indirect constraints based on off-shell measurements [10].

The constraints on the Higgs width obtained from the off-shell measurements are not model-independent; the primary assumption is that the effective Higgs couplings to SM particles do not differ substantially in the on-shell and off-shell regions [11-13]. There are several ways to make this assumption invalid. For example, one can extend the theory to include relatively light colored particles that contribute to the $g g H$ coupling [12], new Higgs resonances [13] or anomalous $H Z Z$ couplings [14-16]. However, all such cases will give rise to relatively clear signatures of New Physics at the LHC, beyond changes in the Higgs width or a change in the number of events in the off-shell region. As the result, the validity of the assumptions crucial for the extraction of the Higgs width from the comparison of off- and on-shell Higgs production cross sections can be experimentally validated.

However, if the couplings of the Higgs boson to gluons and vector bosons are modified, the yield of vector bosons $V$, produced in gluon fusion, changes in a complicated way. This is because the Higgs signal $g g \rightarrow H \rightarrow V V$ amplitudes and their interference with the prompt production amplitudes scale differently. As a result, it is important to investigate properties of the signal $g g \rightarrow H \rightarrow V V$, the irreducible background $g g \rightarrow V V$, and the interference separately. In particular, the QCD corrections to each of these contributions should be known since this information is required to properly simulate the $g g \rightarrow Z Z$ process with modified Higgs couplings.

A significant amount of recent effort has been focused on QCD corrections to both Higgs and massive $V V$ production, resulting in the former being computed to next-tonext-to-next-to leading order $\left(\mathrm{N}^{3} \mathrm{LO}\right)$ in $\mathrm{QCD}$ in the heavy top limit $[17,18]$ and the latter to next-to-next-to leading order (NNLO) [19-23]. By contrast, although the gluonic prompt background (with on-shell $Z$ ) is known through next-to-leading order (NLO) in QCD $[24,25]$, the interference has so far only been computed at leading order (LO). This is unfortunate since the QCD corrections to gluon-induced processes are known to be significant, partially due to the high likelihood of emitting a hard gluon in the processes $g g \rightarrow H$ and $g g \rightarrow V V$. In the current experimental analyses, the QCD enhancement of the interference effects is modeled approximately, by assuming that it is related to the known QCD enhancement of the signal. While this is a plausible hypothesis which can be justified if universal soft QCD radiation provides a dominant source of radiative corrections [26, 27], it is important to verify it by an explicit computation. Such verification as well as the computation of the realistic QCD enhancement factor for the interference accounting for off-shell effects, vector boson decays and fiducial cuts used by ATLAS and CMS collaborations [7-9], become increasingly important since the LHC experiments are posed to push the off-shell measurements to a new level of precision. 
Since both signal and background processes are loop-induced, QCD computations for each of them require two-loop amplitudes. In the case of Higgs production in gluon fusion, the two-loop virtual amplitudes are known since long ago [28-30]. For the background process $g g \rightarrow V V$ the situation is more complex. Indeed, for both neutral and charged vector bosons, $V=Z / \gamma$ and $V=W^{ \pm}$, both massive and massless quarks contribute to the two-loop $g g \rightarrow V V$ amplitude. The massless contributions were computed during the last year [31,32], whereas an explicit computation of massive contributions is currently not feasible. This is because this calculation involves two-loop four-point functions with internal and external massive lines, which, despite the recent success in evaluating these amplitudes for the $g g \rightarrow H H$ process [33], appear to be beyond current loop techniques. For $V=Z / \gamma$, the problem can be circumvented by performing an expansion in the inverse top mass as suggested in ref. [34]. For $V=W$, the expansion in $1 / m_{t}$ can be done along similar lines but it is more complicated since massive (top) and massless (bottom) propagators appear in contributing diagrams at the same time. For this reason we do not compute contributions of third-generation quarks to $g g \rightarrow W^{+} W^{-}$in this paper, leaving it for future work.

The expansion of the scattering amplitude $g g \rightarrow Z Z$ in $1 / m_{t}$ is expected to be valid if the partonic center-of-mass energy is below the top quark pair production threshold, $m_{4 \ell}<2 m_{t}$. This leaves a significant window of energies $2 m_{Z}<m_{4 \ell}<2 m_{t}$ where the interference effects are important and the heavy-top expansion is expected to provide a reasonably accurate approximation to the massive $g g \rightarrow Z Z$ amplitudes. Computation of QCD corrections to the interference for the invariant masses of the two $Z$-bosons larger than $2 m_{t}$ remains an interesting problem; it can only be fully addressed by studying the NLO QCD corrections to $g g \rightarrow Z Z$ amplitudes with the exact mass dependence.

The remainder of this paper is organized as follows. In section 2, we focus on $Z Z$ production in gluon fusion. We discuss details of the calculation, including validation of the $1 / m_{t}$ expansion, and present results applicable to the LHC phenomenology. In section 3, we present the calculation and discuss phenomenology of the $W W$ production in gluon fusion. We conclude in section 4 .

\section{$2 \quad Z Z$ production}

\subsection{Details of the calculation}

Scattering amplitudes for processes $g g \rightarrow Z Z$ and $g g \rightarrow Z Z+g$ can be written as

$$
\mathcal{A}_{\mathrm{ZZ}}=\mathcal{A}_{H}+\mathcal{A}_{p}
$$

where the first amplitude describes the Higgs-mediated signal process $g g \rightarrow H \rightarrow Z Z$ or $g g \rightarrow H \rightarrow Z Z+g$ and the second amplitude describes the "background" prompt production $g g \rightarrow Z Z$ and $g g \rightarrow Z Z+g$. Although not explicit in these notations, the leptonic decays of $Z$-bosons are always included in the calculation and the $Z$-bosons are not assumed to be on the mass shell. For background processes, $\gamma^{*}$-mediated amplitudes are also included. Upon squaring the amplitude in eq. (2.1), one obtains three terms

$$
\left|\mathcal{A}_{Z Z}\right|^{2}=\left|\mathcal{A}_{H}\right|^{2}+\left|\mathcal{A}_{p}\right|^{2}+2 \operatorname{Re}\left[\mathcal{A}_{H}^{*} \mathcal{A}_{p}\right]
$$




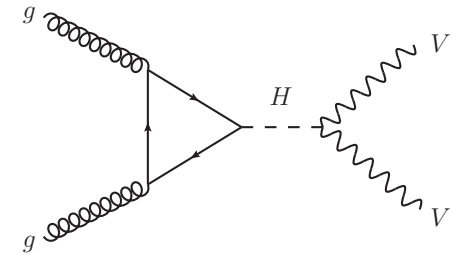

(a)

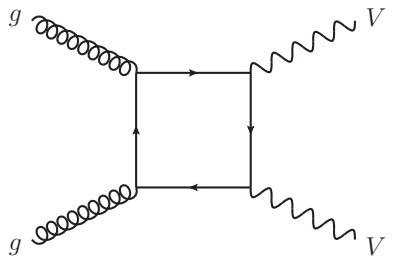

(b)

Figure 1. Representative Feynman diagrams for the Higgs-mediated signal amplitude $g g \rightarrow H \rightarrow$ $Z Z$ (a) and the background amplitude $g g \rightarrow Z Z$ (b) at LO in pQCD. The decays of the $Z$-bosons to leptons are understood.

that, upon integration over the phase-space of the relevant final states, produce the corresponding contributions to the cross section. We will refer to the three contributions to the cross sections, shown in eq. (2.2), as the signal, the background and the interference, respectively. Note that the interference contribution to the cross section is not sign-definite, in contrast to contributions of both the signal and background.

We now describe the ingredients that we use to assemble the full scattering amplitude $\mathcal{A}_{Z Z}$. The one-loop LO amplitudes $\mathcal{A}_{H}$ and $\mathcal{A}_{p}$ are shown in figure 1 . The former, with full dependence on the quark masses that facilitate $g g H$ interaction, has been known for a long time. The latter amplitudes for both massless and massive quark contributions were computed in [35-37]; more recent computations are available in the codes gg2VV [38] and MCFM $[5,39]$. We make use of the amplitudes from MCFM in our calculation.

For the NLO QCD computation we need virtual corrections to $g g \rightarrow Z Z$ and real contributions $g g \rightarrow Z Z+g$ (see examples of contributing diagrams in figure 2). To compute the corresponding scattering amplitudes, we use the expressions for the two-loop contribution to $\mathcal{A}_{H}$ from ref. [30]. The contribution of massless quarks to two-loop background amplitudes $\mathcal{A}_{p}$ has been calculated in refs. [31, 32]. The public libraries of ref. [32] were already used to compute the NLO QCD corrections to the gluon-induced $Z Z$ continuum production [24] and we borrow the relevant amplitude from that reference. The NLO QCD corrections to the contribution of the top loops to $Z Z$ production is not known in an analytic form; we compute it using an expansion in $1 / m_{t}$. The technical details of the calculation are described below.

The amplitudes for real emission contributions are assembled in a similar way. For the signal process $g g \rightarrow H+g \rightarrow Z Z+g$ the amplitude was computed long ago in refs. [40, 41]. For the prompt production process $g g \rightarrow Z Z+g$, amplitudes that describe the contribution of massless quarks were calculated using a combination of analytic and numerical unitarity methods in ref. [24]. The contribution of top quark loops to $g g \rightarrow Z Z+g$ amplitudes is not known analytically; we obtain it as an expansion in $1 / m_{t}$ as described below. Alternatively, these amplitudes can be obtained from one-loop providers [42-46]. For our studies, we often used the OpenLoops program [42] as a cross-check of our implementation.

Since we allow for off-shell production of the $Z$-bosons, we also include single-resonant amplitudes. Amplitudes $g g \rightarrow Z^{*} \rightarrow 4 l$ receive contributions from massless and massive triangle diagrams which vanish at any loop order provided that the gluons are on-shell [47, 48]. 




$(a)$



(c)



(e)

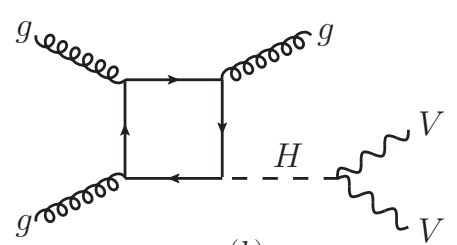

(b)

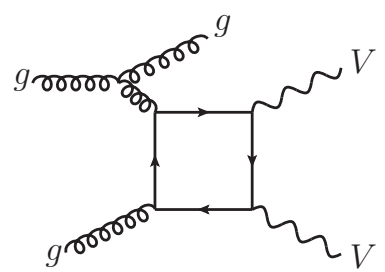

(d)

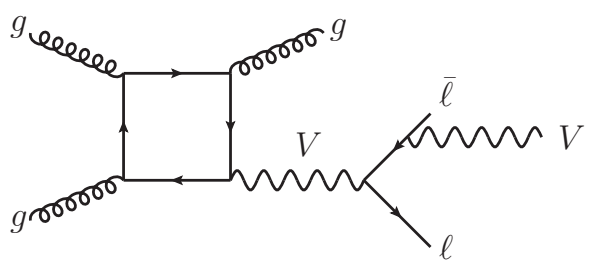

$(f)$

Figure 2. Representative Feynman diagrams at NLO. Shown are the two-loop and real emission contributions to the signal amplitude $\mathcal{A}_{H}\left((\mathrm{a})\right.$ and (b)) and to the background amplitude $\mathcal{A}_{p}((\mathrm{c})-$ (f)). The decays of the $Z$-bosons to leptons are only shown in (f).

For this reason, we only need to consider single-resonant amplitudes with an emitted gluon $g g \rightarrow g Z^{*} \rightarrow g+4 l$ shown in figure $2(\mathrm{f})$; the analytic expressions for amplitudes that contain both massless and massive loops are given in ref. [49]. We checked our implementation of single-resonant amplitudes against the OpenLoops program [42] and found good agreement.

As previously mentioned, the top quark contribution to the two-loop amplitude for $g g \rightarrow Z Z$ prompt production is intractable at present. In order to get around this, we compute this amplitude in a heavy-top expansion, keeping terms up to $\mathcal{O}\left(m_{t}^{-8}\right)$. The calculation employs the standard procedures of the large mass expansion (see e.g. ref. [50]) that allows one to express all contributing diagrams through a linear combination of vacuum bubble integrals and one-loop three-point functions with massless internal lines, which can be easily computed.

We also include massless and massive double-triangle diagrams figure 2(e) that are anomalous and are required to simultaneously account for bottom and top quark contributions. The analytic results for these triangle diagrams can be found in refs. [47, 48]. These diagrams feature a highly off-shell $t$-channel gluon, and consequently only contribute to final results at the level of just a few per mill.

The last amplitude that we need to consider is the top quark contribution to the real emission amplitude for $g g \rightarrow Z Z+g$ prompt production. This one-loop amplitude is not 
known in a closed analytic form. We computed it in the same way as the two-loop virtual amplitude, by expanding in the inverse top quark mass. Similar to the virtual correction, the expansion can be carried out at the level of the amplitude keeping the full dependence on the off-shellness of the $Z$-bosons and allowing for their subsequent decay into a lepton pair. Below we discuss the conditions under which this expansion is valid. For now, it suffices to say that squares of the top-quark induced amplitudes for $g g \rightarrow Z Z+g \rightarrow 4 l+g$ have been checked against the OpenLoops [42] for a number of kinematic point with an unphysically heavy top quark mass, where the $1 / m_{t}$ expansion is expected to work well. Upon doing that, good agreement at the level of $10^{-5}-10^{-6}$ was found. Strictly speaking, one could have used the results from OpenLoops or other one-loop providers to avoid the $1 / m_{t}$ expansion for real emission diagrams. However this is not necessary since, as we will show in the following, the $1 / m_{t}$ expansion works well in the kinematics region we are interested in.

We do not consider contributions to $Z$-pair production caused by $q g$ fusion, $q g \rightarrow Z Z+$ $q$. The contribution of these processes to the interference is expected to be several times smaller than interference effects from the gluon fusion [51]. Moreover, it is not possible to disentangle these contributions from other $q g$ or $\bar{q} g$ contributions to $Z Z$ production that appear already as $\mathcal{O}\left(\alpha_{s}^{3}\right)$ corrections to the main production mechanism $q \bar{q} \rightarrow Z Z$. With this choice, our result is contaminated by non-canceling factorization scale terms which are however suppressed by the ratio of quark to gluon luminosities, i.e. comparable to other terms we neglect in the full $\mathcal{O}\left(\alpha_{s}^{3}\right) q \bar{q} \rightarrow Z Z$ computation. We prefer not to include these terms to avoid artificially small factorization scale uncertainties, although a proper study of their effect is beyond the scope of this work.

We will now discuss a number of checks that validate the implementation of all the amplitudes in our numerical code and the validation of the approximate treatment of top quark mass effects. The implementation of all the various amplitudes in our code was checked extensively by comparing a large number of leading order kinematic distributions with MCFM [5] and by comparing the various one-loop amplitudes against OpenLoops [42]. As we already mentioned, for these checks we often take the top quark mass to have an unphysically large value, to ensure that the mass expansion of the amplitudes converges. Nevertheless, these checks of the implementation still leave as an open question whether the $1 / m_{t}$ expansion of physical cross sections for $Z Z$ production in gluon fusion actually converges.

To investigate this issue, we begin at $\mathrm{LO}$, where we can perform a comparison of exact and expanded in $1 / m_{t}$ contributions to prompt production of $Z$-pairs. Such a comparison is shown in the left pane in figure 3 . We see that the $1 / m_{t}$ expansion works decently all the way up to $m_{4 \ell} \lesssim 320 \mathrm{GeV}$; after that the exact and expanded result show significant differences. We now combine contributions of leading order massless and massive loops to $g g \rightarrow Z Z$ prompt production and show the ensuing $\mathrm{d} \sigma_{p} / \mathrm{d} m_{4 \ell}$ in the right pane of figure 3 . The result clearly demonstrates that contributions of massless loops dominate so strongly that any issues with expansions in $1 / m_{t}$ around the top quark threshold, visible in the left pane, become unobservable. Indeed, below the top threshold the deviations between exact and approximate results seen in figure 3 only affect the total result at the sub-percent level. 

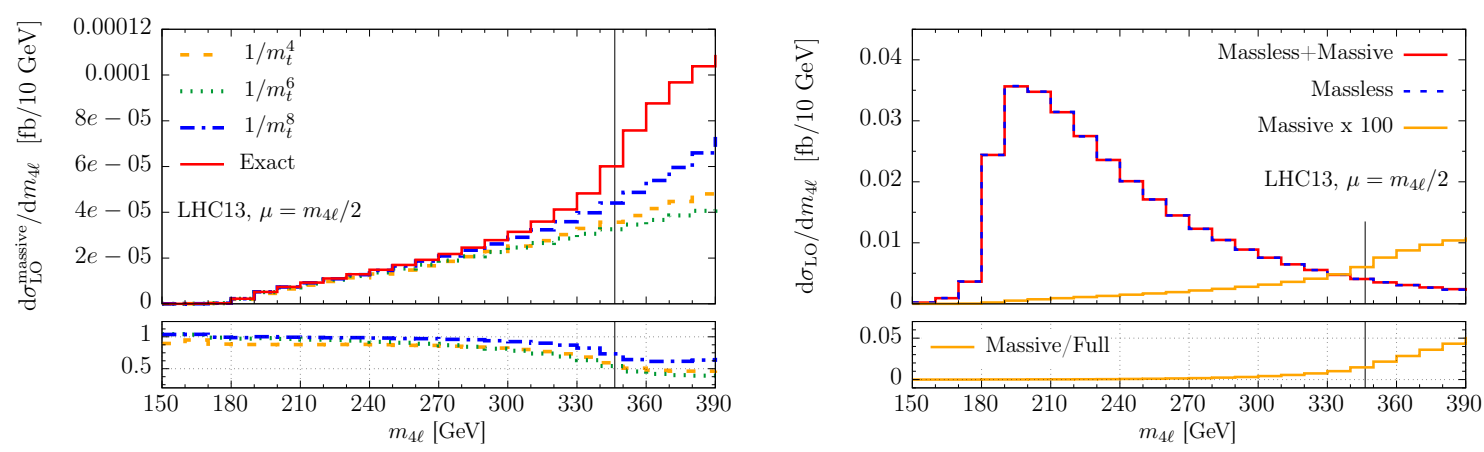

Figure 3. LO invariant mass of the four lepton system at the $13 \mathrm{TeV}$ LHC, background only. In both plots, the vertical line marks the top threshold. Left, upper panel: results using a massive loop only, with the amplitude evaluated in the heavy-top expansion up to various orders in $1 / m_{t}$, compared to the exact mass dependence. The lower panel shows the ratio of the various $1 / m_{t}$ approximations to the exact result. Right, upper panel: distribution using both massless and massive loops compared to massless-only and (exact) massive-only. Note that the latter is multiplied by 100 . The lower panel shows the ratio of the (exact) massive contribution to the full result.



Figure 4. LO results for signal/background interference at the $13 \mathrm{TeV}$ LHC. Both the full result as well as massless/massive-only contributions are shown. Solid line: exact result. Dashed line: $1 / m_{t}$ expansion, including up to $1 / m_{t}^{8}$ terms. The vertical line marks the top threshold.

The situation is however different if one considers the interference between signal and background. Indeed, it is expected on general grounds that top quark contributions to the interference play a much more important role, because for $m_{4 \ell} \geq 2 m_{Z}$, the off-shell Higgs boson decays preferentially to longitudinal $Z$-bosons. In turn, the longitudinal $Z$ bosons have stronger couplings to top quark loops than to massless loops; as a result the contribution of top quark loops is more prominent in the interference than in the background cross section. These expectations are confirmed in figure 4 where we show the interference contribution to the $m_{4 \ell}$ invariant mass distribution. Although the qualitative behavior of massless and massive contributions to the full result is similar to the pure background case - massless/massive contribution decreasing/increasing with $m_{4 \ell}$ - the impact of massive amplitudes is quite sizable. At the top quark threshold $m_{4 \ell} \sim 2 m_{t}$, the two contributions become comparable. At this value of $m_{4 \ell}$, the differences between 
exact and $1 / m_{t}$-expanded results start to appear. Still, it follows from figure 4 , that the error associated with using the $1 / m_{t}$ expansion for the interference is a few percent even at the high end of the expansion region which, as we will see, is smaller than other sources of uncertainty such as uncalculated higher order corrections. We therefore conclude that we can use the heavy top quark mass expansions to study the interference in $g g \rightarrow Z Z$ provided that we restrict ourselves to $m_{4 \ell} \leq 2 m_{t}$.

Since the kinematic features of the virtual corrections are identical to those of leading order amplitudes, the $1 / m_{t}$ expansion of the two-loop amplitude is expected to be valid for $m_{4 \ell}<2 m_{t}$ as well. This is not necessarily the case for the real emission contributions, since a hard gluon emission can resolve the top loop even if $m_{4 \ell}<2 m_{t}$. In order to understand the effect of a hard jet on the $1 / m_{t}$ expansion, we compare our results with those of ref. [51], where the interference effects are calculated in the presence of hard jet. We note that while this calculation includes the full mass dependence in the amplitudes, it only considers on-shell $Z$-bosons whose decays are modeled by multiplying the result of the calculation by the $Z \rightarrow \ell \ell$ branching ratios. For the sake of comparison, we can circumvent these differences by keeping the $Z$-bosons on-shell and integrating over the phase space of the produced leptons. We then compare the $m_{4 \ell}$ distribution of the interference contribution in $g g \rightarrow Z Z+g$ above the $2 m_{Z}$ threshold with the $m_{Z Z}$ distribution of ref. [51]. We find that, in order to have an agreement between expanded and exact results, we need to introduce an upper cut on the transverse momentum of a jet $p_{\perp j}^{\max }$. We need to choose $p_{\perp j}^{\max }$ as large as possible and, at the same time, attempt to maintain the convergence of the $1 / \mathrm{m}_{t}$ expansion. We have found, empirically, that a cut as large as $p_{j, \perp}^{\max }<150 \mathrm{GeV}$ allows us to obtain good agreement with the calculation of ref. [51] all the way up to the top production threshold (see figure 5), while only excluding about $8 \%$ of hard jet events. We will use this cut when we study the LHC phenomenology in the next section. We conclude this section by stressing that in this paper we are mostly interested in genuine NLO corrections to the $g g \rightarrow 4 l$ process. As a consequence, this relatively hard upper cut on jet emission is not particularly relevant for us, since in the region $p_{\perp j}>p_{\perp, j}^{\max }$ our computation is only LO and if desired the result in this region can be obtained using automatic one-loop frameworks, see e.g. $[46,52]$.

\subsection{LHC phenomenology}

In this section, we present the calculation of the NLO QCD corrections to $g g \rightarrow Z Z$ production at the $\sqrt{s}=13 \mathrm{TeV}$ LHC, including off-shell Higgs and the interference effects. For background processes, $\gamma^{*}$-mediated processes are included as well. We employ the following parameters in our computation

$$
\begin{aligned}
m_{Z} & =91.1876 \mathrm{GeV}, & m_{W} & =80.398 \mathrm{GeV}, \\
\Gamma_{Z} & =2.4952 \mathrm{GeV}, & \Gamma_{W} & =2.1054 \mathrm{GeV}, \\
m_{t} & =173.2 \mathrm{GeV}, & G_{F} & =1.16639 \times 10^{-5} \mathrm{GeV}^{-2}, \\
g_{w}^{2} & =4 \sqrt{2} m_{W}^{2} G_{F}, & \sin ^{2} \theta_{W} & =0.2226459 .
\end{aligned}
$$

We use the bottom quark mass $m_{\mathrm{b}}=4.5 \mathrm{GeV}$ when evaluating the amplitude for Higgsmediated processes. However, we take $m_{\mathrm{b}}$ to be massless when computing the amplitudes 


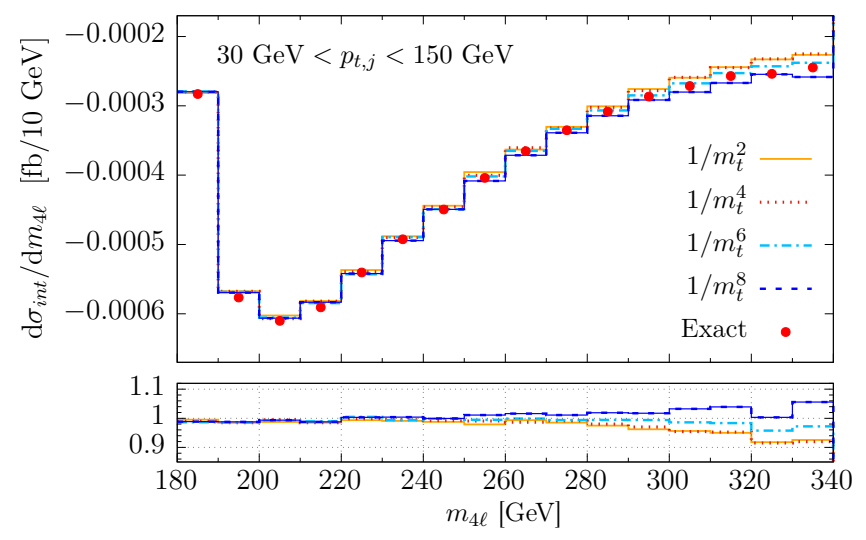

Figure 5. Interference pattern in $g g \rightarrow Z Z+$ jet between the Higgs signal and the prompt production, at the $\sqrt{s}=13 \mathrm{TeV}$ LHC for a scale $\mu=m_{Z Z} / 2$, including both massless and massive contributions. A comparison of expanded in $1 / m_{t}$ and exact results are shown, with the latter taken from ref. [51].

for the prompt production process $g g \rightarrow Z Z$. We use LO and NLO NNPDF3.0 parton distribution functions [53] to obtain leading and next-to-leading order results, respectively. We use dynamical renormalization and factorization scales with the central value $\mu_{0}=$ $m_{4 \ell} / 2$, and vary it by a factor of two in either direction to estimate the scale dependence of the final result.

Apart from the restrictions on $m_{4 \ell}$ and the jet transverse momentum discussed in the previous section, we only impose cuts on the invariant mass of the lepton pairs, $60 \mathrm{GeV}<$ $m_{\ell \ell}<120 \mathrm{GeV}$ to isolate the $Z$ resonance and suppress $\gamma^{*}$ contributions.

We start by considering the $g g \rightarrow Z Z \rightarrow e^{+} e^{-} \mu^{+} \mu^{-}$production in the four-lepton invariant mass interval $150 \mathrm{GeV}<m_{4 \ell}<340 \mathrm{GeV}$. The lower boundary separates Higgs off-shell events from Higgs on-shell events; the upper boundary is imposed to ensure the validity of the $1 / m_{t}$ expansion.

We begin by presenting the results for the cross sections for $g g \rightarrow Z Z \rightarrow e^{+} e^{-} \mu^{+} \mu^{-}$ in the interval of four-lepton invariant masses described above. We show results for the signal, the background, the interference and the full cross section. We find the following results at leading and next-to-leading orders in perturbative QCD

$$
\begin{aligned}
\sigma_{\mathrm{LO}}^{\text {signal }} & =0.043_{-0.009}^{+0.012} \mathrm{fb}, & & \sigma_{\mathrm{NLO}}^{\text {signal }}=0.074_{-0.008}^{+0.008} \mathrm{fb} \\
\sigma_{\mathrm{LO}}^{\text {bkgd }} & =2.90_{-0.58}^{+0.77} \mathrm{fb}, & & \sigma_{\mathrm{NLO}}^{\text {bkgd }}=4.49_{-0.38}^{+0.34} \mathrm{fb} \\
\sigma_{\mathrm{LO}}^{\text {intf }} & =-0.154_{-0.04}^{+0.031} \mathrm{fb}, & & \sigma_{\mathrm{NLO}}^{\text {intf }}=-0.287_{-0.037}^{+0.031} \mathrm{fb} \\
\sigma_{\mathrm{LO}}^{\text {full }} & =2.79_{-0.56}^{+0.74} \mathrm{fb}, & & \sigma_{\mathrm{NLO}}^{\text {full }}=4.27_{-0.35}^{+0.32} \mathrm{fb},
\end{aligned}
$$

where the sub- and superscripts indicate the scale variation. The interference is destructive, as implied by the unitarity arguments, despite the fact that these cross sections refer to the production of four leptons with invariant masses that are far below the values for which the unitarity arguments are valid. Negative interference implies that the physical cross section is smaller than the sum of the signal and background cross sections by about $5 \%$. 

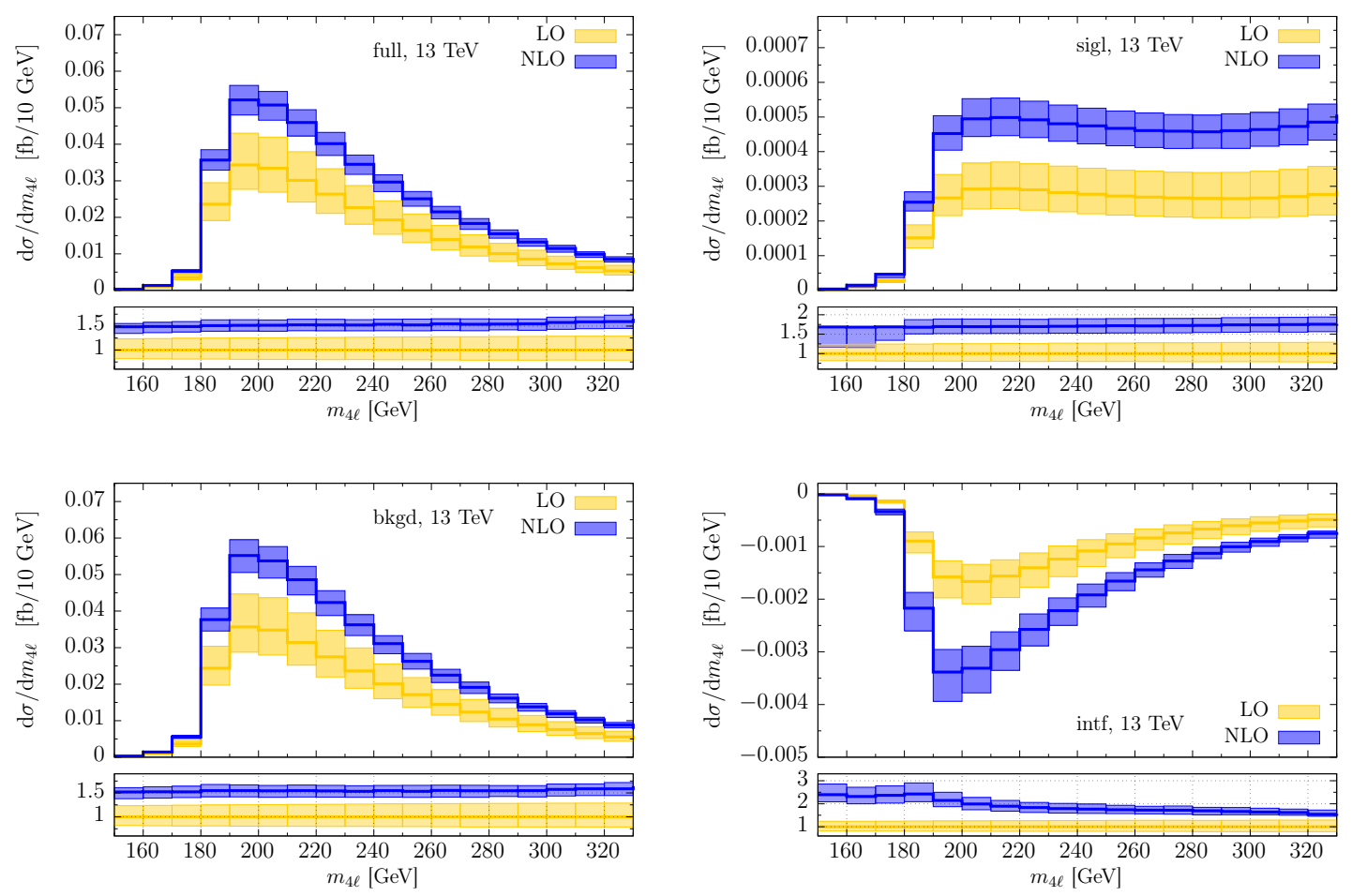

Figure 6. Four-lepton invariant mass distributions in $g g \rightarrow Z Z$ processes at the $13 \mathrm{TeV}$ LHC. The full result is shown as well as contributions of signal, background and interference separately. LO results are shown in yellow, NLO results are shown in blue, and scale variation is shown for $m_{4 \ell} / 4<\mu<m_{4 \ell}$ with a central scale $\mu=m_{4 \ell} / 2$. The lower pane shows the $K$-factors.

We also note that the absolute value of the interference is $3-4$ times larger than the signal, but is still more than an order of magnitude smaller than the irreducible $g g$ background. Consequently, extracting the signal and observing the effect of the interference in this range of four-lepton invariant masses will be challenging, assuming the Higgs couplings to vector bosons and gluons are close to what is expected in the Standard Model.

We observe that the NLO QCD corrections are largest for the signal cross section and smallest for the background. The corresponding $K$-factors ${ }^{1}$ are $K_{\text {signal }}=1.72$ and $K_{\mathrm{bkgd}}=1.55$ for the central scale choice. It is interesting to note that the $K$-factor for the interference, $K_{\text {intf }}=1.65$, is very close to the geometric mean of these results $K_{\text {intf }} \approx \sqrt{K_{\text {bkgd }} K_{\text {signal }}}$, as was assumed in experimental analyses aimed at constraining the Higgs boson width [7,9]. The scale uncertainty of the leading order cross section is in the range of twenty to thirty percent; the NLO cross sections are outside the scale uncertainty of the leading order result. At NLO, the relative scale uncertainty decreases by about a factor of two and becomes close to ten percent.

We continue with the discussion of differential distributions in the invariant mass of four leptons, $m_{4 \ell}$. In figure 6 we show separately the distributions for the signal, the background, the interference and the total yield of four leptons in gluon fusion. The lower

\footnotetext{
${ }^{1}$ We define the $K$-factor as the ratio of NLO corrected cross section at a particular scale to the leading order cross section at the central scale.
} 


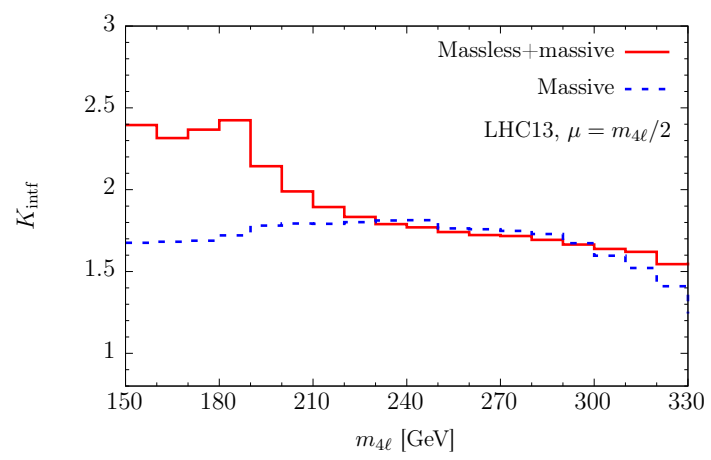

Figure 7. Comparison of full (massive+massless) and massive only interference $K$-factors as a function of $m_{4 \ell}$ at the $13 \mathrm{TeV}$ LHC.

panes show the corresponding $K$-factors, in dependence of $m_{4 \ell}$. We note that $K$-factors for the signal and the background distributions are relatively flat, with a slight increase with $m_{4 \ell}$. The situation with the interference is different. In this case, the $K$-factor around the $2 m_{Z}$ threshold is large, $K_{\text {intf }} \approx 2.5$ for $m_{4 \ell} \lesssim 2 m_{Z}$. As the invariant mass increases, the interference $K$-factor decreases rapidly and flattens out, reaching the value $K_{\text {intf }} \approx 1.5$ at $m_{4 \ell}=2 m_{t}$. Hence, at around $m_{4 \ell} \sim 2 m_{t}$, values of the interference, signal and background $K$-factors become very similar and, practically, independent of the value of the invariant mass $m_{4 \ell}$. Thus, we find that the impact of NLO QCD corrections on the interference $K$-factor can be approximated by the geometric mean of the signal and the background $K$-factors when the interference is integrated over the full kinematic range of four-lepton masses, as well as at higher values of the invariant masses where $K_{\text {signal }} \approx K_{\text {bkgd }} \approx K_{\text {intf }}$. However, this is not the case close to $2 m_{Z}$ threshold, where the behavior of the interference $K$-factor is different from either the signal or background $K$-factors.

Finally, we compare the behavior of the NLO corrections to the interference arising from massive prompt production amplitudes to the full interference result which arises from both massless and massive loops. Such a comparison is shown in figure 7. We have already seen that the massless contribution strongly dominates the interference at around $m_{4 \ell} \sim 2 m_{Z}$ and, as seen from the behavior of the full result, drives a rapid increase in the $K$-factor close to $m_{4 \ell} \sim 2 m_{Z}$. In contrast, $K_{\text {intf }}$ for the massive loops remains flat for $m_{4 \ell} \lesssim 280 \mathrm{GeV}$, at which point it begins to decrease. This means that $K_{\text {intf }}$ for the massive loops is well approximated by the geometric mean $\sqrt{K_{\mathrm{bkgd}} K_{\text {signal }}}$ across the full range of $m_{4 \ell}$ that we consider.

\section{$3 \quad W W$ production}

In this section, we discuss the production of $W$-boson pairs in gluon fusion, including interference effects. Such interference effects have previously been studied at LO in refs. [6, 54]. Unlike in $Z Z$ production, the invariant mass of the off-shell Higgs is not observable, because of neutrinos in the final state. Nevertheless, it is possible to use the transverse mass of the $W W$ system to probe the off-shell Higgs physics. 
The set up of our calculation is similar to the case of $Z$-boson pair production described in the previous section. The principal difference between the two cases is that for $W$-boson pair production, we do not include the contribution of the third generation of quarks when computing QCD radiative corrections. It is known that this contribution amounts to approximately $10 \%$ of the gluonic $W W$ cross section at LO $[54,55]$. As mentioned in the Introduction, this omission is due to the complexity of performing a mass expansion with both top and bottom quarks in the loop. Therefore, our results for $g g \rightarrow W W$ are necessarily incomplete but they, at least, give partial information about radiative effects in the case of the $W W$ production in gluon fusion.

The amplitudes for $g g \rightarrow W W$ production are assembled along the lines described in section 2. We consider leptonic decays of the $W$-bosons, $g g \rightarrow W W \rightarrow \nu_{e} e^{+} \mu^{-} \bar{\nu}_{\mu}$, and consistently include the required single-resonance contributions; this allows us to describe the $W$-pair production for a broad range of invariant masses both below and above the $2 m_{W}$-threshold.

We present results for the $\sqrt{s}=13 \mathrm{TeV}$ LHC, using the same parameters, scales and parton distribution functions as in the previous section. Since we do not use an expansion in $1 / m_{t}$, we no longer require the cut $p_{\perp j}<p_{\perp j}^{\max }$, and we remove this cut from our analysis. We also do not impose any cuts on the final state leptons, so that the results shown in this section are fully inclusive. We stress, however, that our computation can accommodate any cut on final state leptons, missing energy and jet.

We begin with the discussion of the interplay between contributions of the third and the first two generations to the interference at leading order. The results are shown in figure 8 where a comparison is made in dependence of the transverse mass $m_{T, W^{+} W^{-}}$. The transverse mass is defined

$$
m_{\mathrm{T}, \mathrm{WW}}=\sqrt{2 E_{\perp, \mathrm{miss}} p_{\mathrm{T}, \ell \ell}(1-\cos \tilde{\phi})}
$$

where $p_{\perp, \ell \ell}$ is the transverse momentum of the lepton pair, $E_{\perp, \text { miss }}$ is the missing energy, and $\tilde{\phi}$ is the azimuthal angle between the direction of the missing energy and the $\ell^{+} \ell^{-}$ system. It is apparent from figure 8 that, unlike the situation for the $g g \rightarrow W W$ cross section, the massless contributions to the interference do not dominate for any value of $m_{T, W^{+} W^{-}}$[54]. In fact, the two first generations and the third generation give, roughly, comparable contributions to the interference, for $m_{T, W^{+} W^{-}} \leq 200 \mathrm{GeV}$; for higher values of the transverse mass, the contribution of the third generation dominates.

We now turn to the impact of NLO corrections. We consider only contributions from the first two quark generations to the prompt production amplitudes at both LO and NLO, so as to treat the results on an equal footing. At $\sqrt{s}=13 \mathrm{TeV}$, the cross sections are

$$
\begin{aligned}
\sigma_{\mathrm{LO}}^{\text {signal }} & =48.3_{-8.4}^{+10.4} \mathrm{fb}, & & \sigma_{\mathrm{NLO}}^{\text {signal }}=81.0_{-8.2}^{+10.5} \mathrm{fb} \\
\sigma_{\mathrm{LO}}^{\text {bkgd }} & =49.0_{-9.7}^{+12.8} \mathrm{fb}, & & \sigma_{\mathrm{NLO}}^{\text {bkgd }}=74.7_{-6.2}^{+5.5} \mathrm{fb} \\
\sigma_{\mathrm{LO}}^{\text {intf }} & =-2.24_{-0.59}^{+0.44} \mathrm{fb}, & & \sigma_{\mathrm{NLO}}^{\text {intf }}=-4.15_{-0.54}^{+0.47} \mathrm{fb} \\
\sigma_{\mathrm{LO}}^{\text {full }} & =95.0_{-17.6}^{+22.6} \mathrm{fb}, & & \sigma_{\mathrm{NLO}}^{\text {full }}=151.6_{-13.9}^{+15.4} \mathrm{fb} .
\end{aligned}
$$

Similar to $Z Z$ production studied in the previous section, interference is destructive, although less important, reducing the full cross section by about $2 \%-3 \%$. In contrast to 


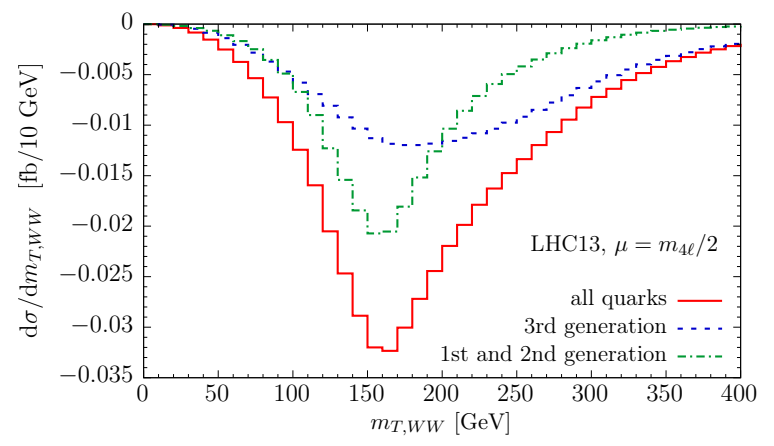

Figure 8. Contributions of the first two generations and the third generation to the interference in $g g \rightarrow W^{+} W^{-}$at leading order in perturbative QCD.

$Z Z$ production, we do not remove the kinematic region corresponding to the Higgs peak, resulting in a signal cross section that is comparable to the background, and more than an order of magnitude greater than the interference.

It is well-understood how to construct cuts to either suppress or enhance the relative contribution of the interference ${ }^{2}$ and we emphasize that, since our computation includes off-shell effects and decays of the $W$-bosons, we can implement any such cuts within our numerical code.

The NLO corrections enhance the signal and background cross sections by $K_{\text {signal }}=$ 1.68 and $K_{\mathrm{bkgd}}=1.53$ respectively, similar to the $K$-values found for $Z Z$ production in the previous section. However, for the interference $K_{\text {intf }}=1.85$, which is larger than the corresponding $K$-factor in $Z$-pair production, $K_{\text {intf }}=1.65$. While the relationship between the interference $K$-factor and the geometric mean $\sqrt{K_{\text {bkgd }} K_{\text {signal }}}$ is no longer exact, the geometric mean still provides a decent approximation to $K_{\text {intf }}$.

We show the $m_{T, W W}$ distributions for the signal, the background, the interference, and the total yield in figure 9 , with the $K$-factors in the lower panes. We note that all LO distributions approach zero for low $m_{T, W W}$, leading to extremely large $K$-factors in this region. Apart from this, $K_{\text {bkgd }}$ is relatively flat, as is $K_{\text {signal }}$ for $m_{T, W W}<2 m_{W}$, after which the signal is suppressed and the statistics are limited. The $K$-factor for the interference again behaves differently, dropping from $K_{\text {intf }} \approx 2$ at $m_{T, W W} \approx 60 \mathrm{GeV}$ to $K_{\text {intf }} \approx 1.5$ at the high end of the distribution. A qualitatively similar effect was seen in the $m_{4 \ell}$ distributions from $Z Z$ production, which again was ascribed to the massless contributions, while the massive contribution remained relatively flat (cf. figure 7). This observation suggests a way of estimating the impact of NLO QCD corrections to the interference including all quark flavors, by adding the NLO results displayed in figure 9 to the LO third generation contribution multiplied by the approximate $K$-factor $\sqrt{K_{\text {bkgd }} K_{\text {signal }}}=1.6$. This procedure results in an approximate NLO interference cross section $\sigma_{\mathrm{NLO} \text {, approx. }}^{\text {intf }}=-8.35 \mathrm{fb}$, to be compared with a $\mathrm{LO}$ result of $\sigma_{\mathrm{LO}}^{\text {intf }}=-4.86 \mathrm{fb}$ including all quark contributions. The corresponding $m_{T, W W}$ distribution is shown in figure 10. Finally, we reiterate that this approximation to the full NLO interference can be improved by calculating the massive

\footnotetext{
${ }^{2}$ See e.g. ref. [8] for a description of experimental selection criteria in off-shell studies.
} 

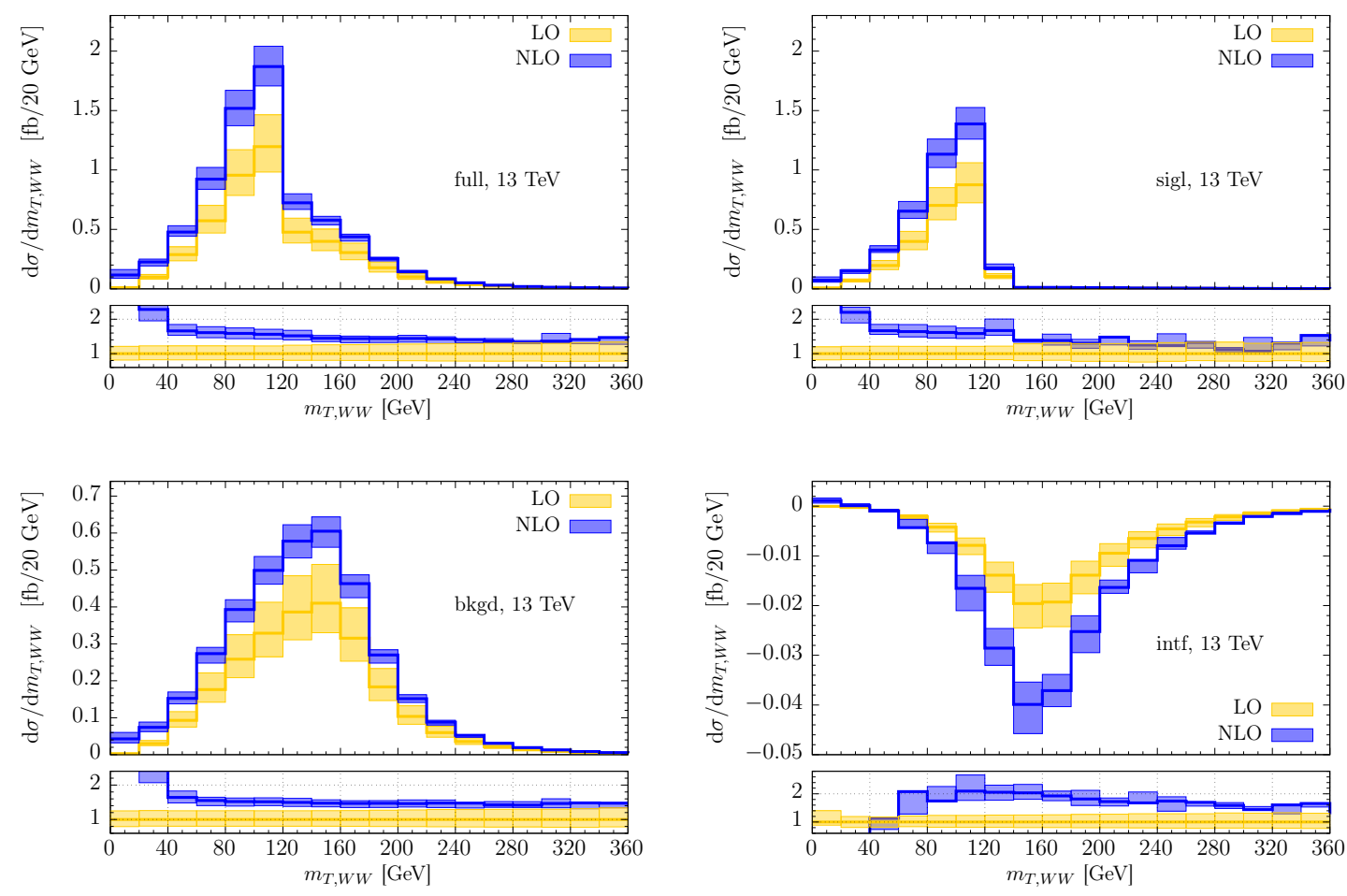

Figure 9. Transverse mass $m_{T, W W}$ distributions in $g g \rightarrow W W$ process at the $13 \mathrm{TeV}$ LHC. The full result is shown as well as contributions of signal, background and interference separately. Only contributions from the first two quark generations to the prompt production amplitudes are included. LO results are shown in yellow, NLO results are shown in blue, and scale variation is shown for $m_{4 \ell} / 4<\mu<m_{4 \ell}$ with a central scale $\mu=m_{4 \ell} / 2$. The lower pane shows the $K$-factors.

loops either in a $1 / m_{t}$ expansion or with the full mass dependence. While the latter is at the limit of our current capabilities, it is the only way in which mass effects can be unambiguously included in all kinematic regimes.

\section{Conclusion}

In this paper, we described the computation of the NLO QCD radiative corrections to the production of four leptons in gluon fusion, $g g \rightarrow V V \rightarrow 4 l, V=Z / \gamma^{*}, W$ and discussed phenomenological implications for the LHC. Our computation includes both prompt and Higgs-mediated production mechanisms, off-shell effects and decays of vector bosons and is fully differential in kinematics variables of final state leptons and jets. Contributions of massive loops are either treated approximately in NLO QCD, as in case of the $Z$-boson pair production, or completely omitted as in the case of $W$-pair production, since the corresponding exact computations are currently not technically feasible. In the case of the $Z$-pair production we construct an expansion of relevant amplitudes in $1 / m_{t}$ and argue that the results of such an expansion can be reliably used for phenomenology provided that the four-lepton invariant mass is restricted to below the $2 m_{t}$ and hard gluons in the final state have transverse momenta below $150 \mathrm{GeV}$. 


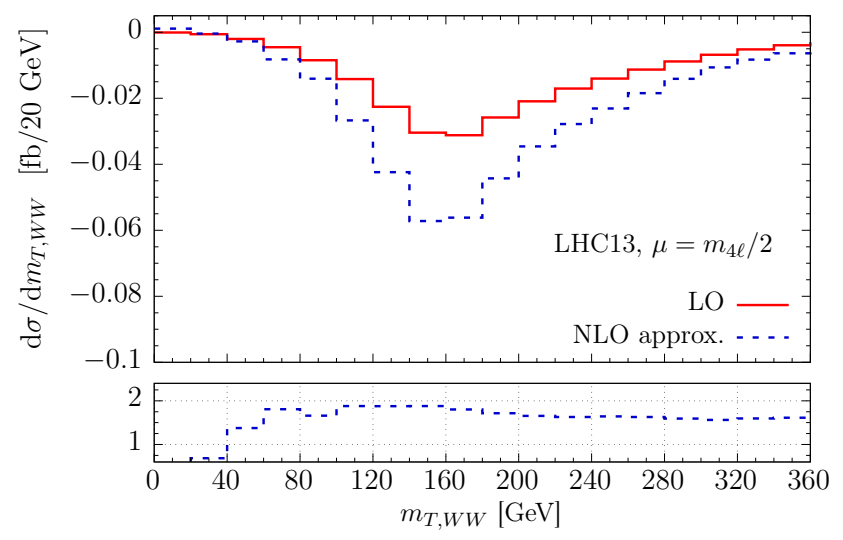

Figure 10. Transverse mass $m_{T, W W}$ distribution for the interference in $g g \rightarrow W W$ at the $13 \mathrm{TeV}$ LHC. The LO result includes contributions from three quark generations. The NLO result is obtained by summing the exact result for the first two generations with the LO third generation contribution multiplied by a constant $K$-factor $\sqrt{K_{\text {bkgd }} K_{\text {signal }}}=1.6$.

We find that the $K$-factors for the interference in $Z$-pair production can be well described as a geometric mean of the $K$-factors for the background and the signal, $K_{\text {intf }} \approx$ $\sqrt{K_{\text {bkgd }} K_{\text {signal }}}$. This relation between the $K$-factors seems to hold both locally and globally, except in the region below and around the $2 m_{Z}$ threshold, where the interference $K$-factor significantly exceeds the $K$-factors for the signal and the background. This feature appears to be driven by the Higgs interference with massless prompt production amplitudes, which dominate the interference in this region.

It is interesting to point out that, in the Higgs signal bin $m_{4 \ell} \sim m_{H}$, the irreducible background $g g \rightarrow Z Z$ is about one percent of the signal while the interference contributes at the level of 0.1 percent. Since the irreducible background is flat across the signal bin, it can be constrained experimentally from side bands. At the same time, since the cross section for Higgs boson production in gluon fusion is currently computed with a few percent precision, the interference contribution needs to receive a $K$-factor of more than 10 to become relevant. Given that the NLO interference $K$-factor stays close to $K \sim 2.5$ below the $2 m_{Z}$ threshold, the required enhancement is highly improbable.

For $W W$ production, we compute the QCD corrections to the interference taking into account the first two (massless) quark generations. We find that the interference $K$-factor in that case is larger than $K$-factors for both the signal and the background. We note, however, that this result is incomplete since for $W W$ production the contribution of top and bottom quarks to the interference is significant. Computation of NLO QCD corrections to the $g g \rightarrow W W$ amplitude for massive internal quarks is an interesting challenge that we leave for future investigation.

\section{Acknowledgments}

We are grateful to S. Pozzorini and J. Lindert for helping us with checks against OpenLoops [42]. The research reported in this paper is partially supported by BMBF 
grant 05H15VKCCA. F.C. and K.M. wish to thank the Kavli Institute for Theoretical Physics for hospitality while part of this work was carried out.

Note added. When this paper was being written, ref. [56] appeared where the NLO QCD corrections to the interference in $g g \rightarrow Z Z$ were studied as well. In contrast to our paper, in ref. [56] the production of on-shell $Z$-bosons above the threshold is studied and their decays are not considered. At the same time, the attempt is made in ref. [56] to extrapolate the $1 / m_{t}$-expansion above the $2 m_{t}$ threshold using conformal mapping and Padé approximants. The results of ref. [56] suggest a close relation between $K$-factors of the signal and the interference other than in the region around the $2 m_{Z}$ threshold, in qualitative agreement with what we observe in this paper.

Open Access. This article is distributed under the terms of the Creative Commons Attribution License (CC-BY 4.0), which permits any use, distribution and reproduction in any medium, provided the original author(s) and source are credited.

\section{References}

[1] ATLAS collaboration, Observation of a new particle in the search for the Standard Model Higgs boson with the ATLAS detector at the LHC, Phys. Lett. B 716 (2012) 1 [arXiv: 1207.7214] [INSPIRE].

[2] CMS collaboration, Observation of a new boson at a mass of $125 \mathrm{GeV}$ with the CMS experiment at the LHC, Phys. Lett. B 716 (2012) 30 [arXiv:1207.7235] [INSPIRE].

[3] N. Kauer and G. Passarino, Inadequacy of zero-width approximation for a light Higgs boson signal, JHEP 08 (2012) 116 [arXiv:1206.4803] [INSPIRE].

[4] F. Caola and K. Melnikov, Constraining the Higgs boson width with ZZ production at the LHC, Phys. Rev. D 88 (2013) 054024 [arXiv: 1307.4935] [INSPIRE].

[5] J.M. Campbell, R.K. Ellis and C. Williams, Bounding the Higgs width at the LHC using full analytic results for $g g \rightarrow e^{-} e^{+} \mu^{-} \mu^{+}$, JHEP 04 (2014) 060 [arXiv: 1311.3589] [INSPIRE].

[6] J.M. Campbell, R.K. Ellis and C. Williams, Bounding the Higgs width at the LHC: Complementary results from $H \rightarrow W W$, Phys. Rev. D 89 (2014) 053011 [arXiv:1312.1628] [INSPIRE].

[7] CMS collaboration, Constraints on the Higgs boson width from off-shell production and decay to Z-boson pairs, Phys. Lett. B 736 (2014) 064 [arXiv:1405.3455] [INSPIRE].

[8] ATLAS collaboration, Constraints on the off-shell Higgs boson signal strength in the high-mass $Z Z$ and $W W$ final states with the ATLAS detector, Eur. Phys. J. C 75 (2015) 335 [arXiv: 1503.01060] [INSPIRE].

[9] CMS collaboration, Limits on the Higgs boson lifetime and width from its decay to four charged leptons, Phys. Rev. D 92 (2015) 072010 [arXiv:1507.06656] [INSPIRE].

[10] CMS collaboration, Properties of the observed Higgs-like resonance using the diphoton channel, CMS-PAS-HIG-13-016 (2013) [INSPIRE].

[11] C. Englert and M. Spannowsky, Limitations and Opportunities of Off-Shell Coupling Measurements, Phys. Rev. D 90 (2014) 053003 [arXiv: 1405.0285] [INSPIRE]. 
[12] C. Englert, Y. Soreq and M. Spannowsky, Off-Shell Higgs Coupling Measurements in BSM scenarios, JHEP 05 (2015) 145 [arXiv: 1410.5440] [INSPIRE].

[13] H.E. Logan, Hiding a Higgs width enhancement from off-shell $g g\left(\rightarrow h^{*}\right) \rightarrow Z Z$ measurements, Phys. Rev. D 92 (2015) 075038 [arXiv:1412.7577] [INSPIRE].

[14] I. Anderson et al., Constraining anomalous HVV interactions at proton and lepton colliders, Phys. Rev. D 89 (2014) 035007 [arXiv:1309.4819] [InSPIRE].

[15] J.S. Gainer, J. Lykken, K.T. Matchev, S. Mrenna and M. Park, Geolocating the Higgs Boson Candidate at the LHC, Phys. Rev. Lett. 111 (2013) 041801 [arXiv:1304.4936] [INSPIRE].

[16] J.S. Gainer, J. Lykken, K.T. Matchev, S. Mrenna and M. Park, Beyond Geolocating: Constraining Higher Dimensional Operators in $H \rightarrow 4 \ell$ with Off-Shell Production and More, Phys. Rev. D 91 (2015) 035011 [arXiv:1403.4951] [INSPIRE].

[17] C. Anastasiou, C. Duhr, F. Dulat, F. Herzog and B. Mistlberger, Higgs Boson Gluon-Fusion Production in QCD at Three Loops, Phys. Rev. Lett. 114 (2015) 212001 [arXiv:1503. 06056] [INSPIRE].

[18] C. Anastasiou et al., High precision determination of the gluon fusion Higgs boson cross-section at the LHC, JHEP 05 (2016) 058 [arXiv: 1602.00695] [INSPIRE].

[19] F. Cascioli et al., ZZ production at hadron colliders in NNLO QCD, Phys. Lett. B 735 (2014) 311 [arXiv:1405.2219] [INSPIRE].

[20] T. Gehrmann et al., $W^{+} W^{-}$Production at Hadron Colliders in Next to Next to Leading Order QCD, Phys. Rev. Lett. 113 (2014) 212001 [arXiv:1408.5243] [INSPIRE].

[21] M. Grazzini, S. Kallweit and D. Rathlev, ZZ production at the LHC: fiducial cross sections and distributions in NNLO QCD, Phys. Lett. B 750 (2015) 407 [arXiv:1507.06257] [INSPIRE].

[22] M. Grazzini, S. Kallweit, D. Rathlev and M. Wiesemann, $W^{ \pm} Z$ production at hadron colliders in NNLO QCD, arXiv:1604.08576 [INSPIRE].

[23] M. Grazzini, S. Kallweit, S. Pozzorini, D. Rathlev and M. Wiesemann, $W^{+} W^{-}$production at the LHC: fiducial cross sections and distributions in NNLO QCD, arXiv:1605.02716 [INSPIRE].

[24] F. Caola, K. Melnikov, R. Röntsch and L. Tancredi, QCD corrections to ZZ production in gluon fusion at the LHC, Phys. Rev. D 92 (2015) 094028 [arXiv:1509.06734] [INSPIRE].

[25] F. Caola, K. Melnikov, R. Röntsch and L. Tancredi, QCD corrections to $W^{+} W^{-}$production through gluon fusion, Phys. Lett. B 754 (2016) 275 [arXiv:1511.08617] [INSPIRE].

[26] M. Bonvini, F. Caola, S. Forte, K. Melnikov and G. Ridolfi, Signal-background interference effects for $g g \rightarrow H \rightarrow W^{+} W^{-}$beyond leading order, Phys. Rev. D 88 (2013) 034032 [arXiv: 1304.3053] [INSPIRE].

[27] C.S. Li, H.T. Li, D.Y. Shao and J. Wang, Soft gluon resummation in the signal-background interference process of $g g\left(\rightarrow h^{*}\right) \rightarrow Z Z$, JHEP 08 (2015) 065 [arXiv: 1504.02388] [INSPIRE].

[28] M. Spira, A. Djouadi, D. Graudenz and P.M. Zerwas, Higgs boson production at the LHC, Nucl. Phys. B 453 (1995) 17 [hep-ph/9504378] [inSPIRE].

[29] R. Harlander and P. Kant, Higgs production and decay: Analytic results at next-to-leading order QCD, JHEP 12 (2005) 015 [hep-ph/0509189] [INSPIRE]. 
[30] U. Aglietti, R. Bonciani, G. Degrassi and A. Vicini, Analytic Results for Virtual QCD Corrections to Higgs Production and Decay, JHEP 01 (2007) 021 [hep-ph/0611266] [INSPIRE].

[31] F. Caola, J.M. Henn, K. Melnikov, A.V. Smirnov and V.A. Smirnov, Two-loop helicity amplitudes for the production of two off-shell electroweak bosons in gluon fusion, JHEP $\mathbf{0 6}$ (2015) 129 [arXiv: 1503.08759] [INSPIRE].

[32] A. von Manteuffel and L. Tancredi, The two-loop helicity amplitudes for $g g \rightarrow V_{1} V_{2} \rightarrow 4$ leptons, JHEP 06 (2015) 197 [arXiv:1503.08835] [INSPIRE].

[33] S. Borowka et al., Higgs Boson Pair Production in Gluon Fusion at Next-to-Leading Order with Full Top-Quark Mass Dependence, Phys. Rev. Lett. 117 (2016) 012001 [arXiv: 1604.06447] [INSPIRE].

[34] K. Melnikov and M. Dowling, Production of two Z-bosons in gluon fusion in the heavy top quark approximation, Phys. Lett. B 744 (2015) 43 [arXiv:1503.01274] [INSPIRE].

[35] E.W.N. Glover and J.J. van der Bij, Z-boson pair production via gluon fusion, Nucl. Phys. B 321 (1989) 561 [INSPIRE].

[36] T. Matsuura and J.J. van der Bij, Characteristics of leptonic signals for $Z$ boson pairs at hadron colliders, Z. Phys. C 51 (1991) 259 [INSPIRE].

[37] C. Zecher, T. Matsuura and J.J. van der Bij, Leptonic signals from off-shell Z boson pairs at hadron colliders, Z. Phys. C 64 (1994) 219 [hep-ph/9404295] [InSPIRE].

[38] T. Binoth, N. Kauer and P. Mertsch, Gluon-induced QCD corrections to $p p \rightarrow Z Z \rightarrow l \bar{\ell} \ell^{\prime} \bar{\ell}^{\prime}$, in proceedings of the 16th International Workshop on Deep Inelastic Scattering and Related Subjects (DIS 2008), London, England, April 7-11 2008, p. 142 [arXiv: 0807.0024] [INSPIRE] and online pdf version at https://inspirehep.net/record/789541/files/arXiv:0807.0024.pdf.

[39] J.M. Campbell, R.K. Ellis and C. Williams, Vector boson pair production at the LHC, JHEP 07 (2011) 018 [arXiv: 1105.0020] [INSPIRE].

[40] R.K. Ellis, I. Hinchliffe, M. Soldate and J.J. van der Bij, Higgs Decay to $\tau^{+} \tau^{-}$: A Possible Signature of Intermediate Mass Higgs Bosons at the SSC, Nucl. Phys. B 297 (1988) 221 [INSPIRE].

[41] U. Baur, E.W.N. Glover and J.J. van der Bij, Hadronic Production of Electroweak Vector Boson Pairs at Large Transverse Momentum, Nucl. Phys. B 318 (1989) 106 [INSPIRE].

[42] F. Cascioli, P. Maierhöfer and S. Pozzorini, Scattering Amplitudes with Open Loops, Phys. Rev. Lett. 108 (2012) 111601 [arXiv:1111.5206] [INSPIRE].

[43] V. Hirschi, R. Frederix, S. Frixione, M.V. Garzelli, F. Maltoni and R. Pittau, Automation of one-loop QCD corrections, JHEP 05 (2011) 044 [arXiv:1103.0621] [INSPIRE].

[44] S. Actis, A. Denner, L. Hofer, A. Scharf and S. Uccirati, Recursive generation of one-loop amplitudes in the Standard Model, JHEP 04 (2013) 037 [arXiv:1211.6316] [INSPIRE].

[45] G. Cullen et al., GoSAm-2.0: a tool for automated one-loop calculations within the Standard Model and beyond, Eur. Phys. J. C 74 (2014) 3001 [arXiv:1404.7096] [INSPIRE].

[46] V. Hirschi and O. Mattelaer, Automated event generation for loop-induced processes, JHEP 10 (2015) 146 [arXiv:1507.00020] [INSPIRE].

[47] K. Hagiwara, T. Kuruma and Y. Yamada, Three jet distributions from the one loop Zgg vertex at $e^{+} e^{-}$colliders, Nucl. Phys. B 358 (1991) 80 [INSPIRE]. 
[48] J.M. Campbell, R.K. Ellis and G. Zanderighi, Next-to-leading order predictions for $W W+1$ jet distributions at the LHC, JHEP 12 (2007) 056 [arXiv:0710.1832] [INSPIRE].

[49] J.J. van der Bij and E.W.N. Glover, Z Boson Production and Decay via Gluons, Nucl. Phys. B 313 (1989) 237 [INSPIRE].

[50] V.A. Smirnov, Applied Asymptotic Expansions in Momenta and Masses, Springer Tracts Mod. Phys. 177 (2002) 1 [inSPIRE].

[51] J.M. Campbell, R.K. Ellis, E. Furlan and R. Röntsch, Interference effects for Higgs boson mediated Z-pair plus jet production, Phys. Rev. D 90 (2014) 093008 [arXiv:1409.1897] [INSPIRE].

[52] F. Cascioli, S. Höche, F. Krauss, P. Maierhöfer, S. Pozzorini and F. Siegert, Precise Higgs-background predictions: merging NLO QCD and squared quark-loop corrections to four-lepton + 0, 1 jet production, JHEP 01 (2014) 046 [arXiv:1309.0500] [INSPIRE].

[53] NNPDF collaboration, R.D. Ball et al., Parton distributions for the LHC Run II, JHEP 04 (2015) 040 [arXiv: 1410.8849] [INSPIRE].

[54] J.M. Campbell, R.K. Ellis and C. Williams, Gluon-Gluon Contributions to $W^{+} W^{-}$ Production and Higgs Interference Effects, JHEP 10 (2011) 005 [arXiv:1107.5569] [INSPIRE].

[55] N. Kauer, Inadequacy of zero-width approximation for a light Higgs boson signal, Mod. Phys. Lett. A 28 (2013) 1330015 [arXiv:1305.2092] [INSPIRE].

[56] J.M. Campbell, R.K. Ellis, M. Czakon and S. Kirchner, Two Loop Correction to Interference in $g g \rightarrow Z Z$, arXiv:1605.01380 [INSPIRE]. 